\title{
Membrane mixing and dynamics in hybrid POPC/PBd-PEO lipid/block co-polymer giant vesicles
}

\author{
Rashmi Seneviratne ${ }^{\text {a }}$, Michael Rappolt ${ }^{\mathrm{b}}$, Lars. J. C. Jeuken $\uparrow^{\mathrm{c}}$ and \\ Paul. A. Beales*a
}
a. School of Chemistry and Astbury Centre for Structural Molecular Biology, University of Leeds, Leeds, LS2 9JT, UK. *Email: p.a.beales@leeds.ac.uk
b. School of Food Science and Nutrition, University of Leeds, Leeds, LS2 9JT UK.
c. School of Biomedical Sciences and Astbury Centre for Structural Molecular Biology, University of Leeds, Leeds, LS2 9JT, UK.
$\dagger$ Present address: Leiden Institute of Chemistry, Leiden University, The Netherlands.




\begin{abstract}
Lipids and block copolymers can individually self-assemble into vesicles, each with their own particular benefits and limitations. Combining polymers with lipids allows for further optimisation of the vesicle membranes for bionanotechnology applications. Here, POPC lipid is mixed with poly(1,2butadiene-block-ethylene oxide) of two different molecular weights $\left(\mathrm{PBd}_{22}-\mathrm{PEO}_{14}, \mathrm{Mr}=1800 \mathrm{~g} \cdot \mathrm{mol}^{-1}\right.$ and $\left.\mathrm{PBd}_{12}-\mathrm{PEO}_{11}, \mathrm{Mr}=1150 \mathrm{~g} \cdot \mathrm{mol}^{-1}\right)$ in order to investigate, how increasing the polymer fraction affects membrane mixing, hydration and fluidity. Intensity contributions of fluorescently labelled lipid and polymer within mixed GUV membranes confirm membrane homogeneity within the hybrids. General polarisation measurements of Laurdan in GUVs showed little change in membrane hydration as polymer fraction is increased, which suggests good structurally compatibility between lipids and polymers that gives rise to well-mixed vesicles. Membrane fluidity in hybrid GUVs was found to decrease non-linearly with increasing polymer fraction. However, the diffusion coefficients for the fluorescent polymer in hybrid membranes did not change significantly with increasing polymer content. While increasing the polymer fraction does reduce the movement of lipids through a polymer-rich matrix, insignificant difference in diffusion coefficients of the polymer suggests that its diffusion is minimally affected by increasing lipid composition in the range studied. These results lay further foundations for the wider development of hybrid vesicles with controlled properties for advanced biotechnologies.
\end{abstract}




\section{Introduction}

Lipids and amphiphilic block co-polymers are common materials for fabrication of synthetic membranes, in the form of lipid vesicles and polymersomes, respectively. ${ }^{1}$ Although lipids are biocompatible, the major drawback of liposomes is their rapid aging caused by the formation of transitory pores resulting in high permeability and their labile structure can lead to low mechanical stability and, eventually, aggregation..$^{2-4}$ Polymer membranes are more colloidally stable and robust and, in general, can be designed to have low permeability. ${ }^{5}$ While pure lipid or polymer systems have successfully been used to demonstrate biotechnological applications of vesicles, blending of these constituents into hybrid structures offers the potential for greater tuneability with the ambition of synergistically combining their best features. Therefore, hybrid lipid-polymer vesicles aim to take advantage of the robustness and chemical versatility of a polymer membrane as well as the biocompatibility and softness provided by a lipid bilayer. ${ }^{6,7}$

While a variety of block copolymer chemical structures have been blended with phospholipids, polybutadiene-poly(ethylene oxide) (PBd-PEO) block copolymers are one of the most commonly studied examples. ${ }^{8-16} \mathrm{PBd}-\mathrm{PEO}$ containing hybrid vesicles may have potential applications in nanomedicine ${ }^{17}$ and the augmentation of membrane proteins for biotechnology applications such as targeted drug delivery, sensors or artificial cells. ${ }^{8,16,18}$

Focusing on membrane protein biotechnology, $\mathrm{PBd}_{22}-\mathrm{PEO}_{14}$ has been blended with POPC lipids for the reconstitution of the membrane enzyme cytochrome $b_{3}$; all hybrid compositions studied were found to maintain $>40 \%$ of their initial reconstituted protein activity after 41 days storage at $4{ }^{\circ} \mathrm{C}$, a significant improvement over purely lipid vesicles. ${ }^{8}$ Further study revealed that $50 / 50 \mathrm{~mol} \% \mathrm{PBd}_{22}-$ $\mathrm{PEO}_{14} / \mathrm{POPC}$ hybrid vesicles retain $20 \%$ of their initial activity after 500 days storage, where the rate of decay in enzyme activity is approximately 5 times slower than for proteoliposomes. ${ }^{19}$ Other membrane proteins have been successfully reconstituted using PBd-PEO hybrid vesicles. $\mathrm{PBd}_{22}-$ $\mathrm{PEO}_{14}$ blended with E.coli extracted lipids has been used to successfully reconstitute two ATP binding cassette membrane proteins, P-gp or NaAtm1. ${ }^{9}$ Combinations of membrane proteins have also been successfully reconstituted into PBd-PEO hybrid vesicles: $\mathrm{F}_{0}-\mathrm{F}_{1}$ ATP synthase and bacteriorhodopsin in $50 \mathrm{~mol} \% \mathrm{PBd}_{22}-\mathrm{PEO}_{14} / \mathrm{POPC}$ hybrid vesicles retained $>50 \%$ of their activity after 42 days. ${ }^{10}$ Besides enhanced durability of protein function, block copolymers have been shown to enhance membrane protein folding into hybrid vesicles: up to $25 \mathrm{~mol} \%$ of $\mathrm{PBd}_{22}-\mathrm{PEO}_{14}$ or $\mathrm{PBd}_{12^{-}}$ $\mathrm{PEO}_{9}$ in DOPC hybrid vesicles enhanced MScL protein folding during cell-free expression. ${ }^{20}$ Therefore improved understanding of the physicochemical properties of these hybrid membranes should further enhance their optimisation for membrane protein biotechnology.

Hybrid vesicles can either form well-mixed, homogeneous membranes or textured membranes with coexisting lateral domains, providing local environments with distinct properties. ${ }^{6,12,13,15,21}$ Phase separation can be tuned depending on the lipid/polymer blend, temperature-dependent phase transitions of these components, the polymer architecture, degree of polymerisation and polymer molecular weight. Early work on the properties hybrid PBd-PEO giant unilamellar vesicles (GUVs) investigated the large $\mathrm{PBd}_{46}-\mathrm{PEO}_{30}$ polymer mixed with POPC. ${ }^{13,14}$ Mixing between these components was not ideal at low lipid composition, where it was assumed that a high energy cost for insertion of these larger polymers into a thin lipid membrane frustrated hybrid vesicle formation, and no GUVs were observed at intermediate compositions. ${ }^{13}$ The GUV membranes that were formed in this study were, however, homogeneous, but biotinylation of either the lipid or polymer and crosslinking by addition of the multivalent neutravidin protein did result in microscopic domain formation. The domain pattern depended on whether the polymer or lipid was biotinylated; polymer cross-linking resulted in a large single domain, while lipid cross-linking gave many small, lipid-rich domains within a polymer matrix. Further work investigating $\mathrm{PBd}_{46}-\mathrm{PEO}_{30}$ hybrid vesicles with saturated lipids, with and without cholesterol, also demonstrated that phase transitions of the lipid components to liquid 
ordered or gel phases could also drive demixing of coexisting domains in hybrid GUVs; domain sizes could be controlled by the cooling rate into the phase coexistence region from an initial well-mixed membrane at higher temperature. ${ }^{14}$ Phase separation has also been reported for hybrid $\mathrm{PBd}_{43}-$ $\mathrm{PEO}_{20} / \mathrm{DPPC}_{\text {GUVs. }}{ }^{15}$

Flow cytometry analysis of nanoscale hybrid vesicles has shown that using the shorter $\mathrm{PBd}_{22}-\mathrm{PEO}_{14}$ polymer produces a much greater (near 100\%) yield of well-mixed hybrid vesicles when blended with POPC, compared to using the larger $\mathrm{PBd}_{46}-\mathrm{PEO}_{30}$ polymer. ${ }^{16}$ GUVs with shorter block copolymers can also form heterogeneous membranes, as seen for $\mathrm{PBd}_{11} \mathrm{PEO}_{8} / \mathrm{DPPC}$ mixtures, where the DPPC lipids form an ordered gel phase. ${ }^{12}$

To aid the development of hybrid vesicles for biotechnology applications, further insight into their mixing and properties are required. Here we focus on shorter PBd-PEO block copolymers $\left(\mathrm{PBd}_{22}-\right.$ $\mathrm{PEO}_{14}, \mathrm{MW}=1800 \mathrm{~g} \cdot \mathrm{mol}^{-1}$ and $\mathrm{PBd}_{12}-\mathrm{PEO}_{11}, \mathrm{MW}=1150 \mathrm{~g} \cdot \mathrm{mol}^{-1}$ ) that have shown potential for incorporation of membrane proteins in mixtures with the lipid POPC, which forms fluid phases at ambient temperature and is therefore more favourable for formation of well-mixed, homogeneous membranes. Furthermore, good mixing between the PBd-PEO polymers and POPC might be expected due to a similar Hildebrand solubility parameter of the PBd block $\left(15.9\left(\mathrm{Jcm}^{-3}\right)^{1 / 2}\right)$ and the alkane tails of lipids like POPC (approx. 14-16 $\left.\left(\mathrm{Jcm}^{-3}\right)^{1 / 2}\right){ }^{22,23}$

In this study, we focus on the mixing and fluidity of hybrid GUVs utilising a lipid-like fluorescent probe and a fluorescently tagged block copolymer. Fluorescence recovery after photobleaching (FRAP) has previously been used to monitor membrane fluidity and viscosity for $\mathrm{POPC} / \mathrm{PBd}_{46}-\mathrm{PEO}_{30}$ GUVs, where the diffusion constant of a lipid probe decreased by almost two orders of magnitude between the pure lipid and pure polymer compositions. ${ }^{13}$ However the impact of shorter PBd-PEO block copolymers on the fluidity of hybrid membranes is yet to be determined. To complement FRAP results, Laurdan, a membrane probe that is sensitive to the molecular packing and hydration state of the membrane, which has a strong influence on membrane fluidity, is also used to investigate the structural properties of these hybrid vesicles. While Laurdan has been employed to study the bulk properties of nanoscale $\mathrm{PBd}_{22}-\mathrm{PEO}_{14} / \mathrm{POPC}$ vesicles, ${ }^{12}$ it has not been studied with microscale spatial resolution, which might reveal heterogeneities between or within individual vesicles. Our results indicate that no phase separation occurred in our hybrid lipid/polymer blends, and that there is no statistically significant difference in membrane order between $\mathrm{PBd}_{22}-\mathrm{PEO}_{14}$ and $\mathrm{PBd}_{12}-\mathrm{PEO}_{11}$ hybrid vesicles. Membrane fluidity in both $\mathrm{PBd}_{22}-\mathrm{PEO}_{14} / \mathrm{POPC}$ and $\mathrm{PBd}_{12}-\mathrm{PEO}_{11} / \mathrm{POPC}$ hybrid GUVs was found to decrease non-linearly with increasing polymer fraction.

\section{Results \& Discussion}

\section{Hybrid GUV membranes are homogenous but displaying a broad composition distribution}

GUVs composed of mixtures of the lipid POPC with either $\mathrm{PBd}_{22}-\mathrm{PEO}_{14}$ or $\mathrm{PBd}_{12}-\mathrm{PEO}_{11}$ were created by using the electroformation method. Hybrid vesicles with $25 \mathrm{~mol} \%, 50 \mathrm{~mol} \%$ and $75 \mathrm{~mol} \%$ polymer were studied by confocal fluorescence microscopy. Fluorescent lipid and polymer probes were included within these vesicle mixtures for imaging ( 2 mol\% of the lipid-like 3,3'dioctadecyloxacarbocyanine perchlorate (DiO) dye and $10 \mathrm{~mol} \%$ of tetramethyl rhodamine labelled polymer, $\left.\mathrm{PBd}_{22}-\mathrm{PEO}_{14}-\mathrm{TMR}\right)$. GUVs were successfully formed at all compositions; the majority of GUVs were unilamellar, with some multilamellar and intralumenal vesicles also observed. Quantitative analysis of GUV populations focused on the unilamellar GUVs. At all hybrid compositions, the vesicle membranes appeared homogenous with no phase separation evident due to preferential partitioning of the lipid or polymer probes into domains of distinct composition (Figure 
1). This is consistent with the observation of homogeneous PBd-PEO/POPC hybrid vesicles across a range of polymer lengths in previous studies. ${ }^{13,16}$

While blending POPC lipid with either $\mathrm{PBd}_{22}-\mathrm{PEO}_{14}$ or $\mathrm{PBd}_{12}-\mathrm{PEO}_{11}$ polymers might be anticipated to form homogenous hybrid membranes, considering the similar Hildebrand solubilities of the hydrophobic blocks, other energetic factors such as hydrophobic mismatch due to differences in preferred membrane thickness, or kinetic factors in the assembly pathway of the vesicles, could lead to compositional heterogeneities in these samples. To investigate this and verify that hybrid vesicles are indeed formed, the relative intensities of a fluorescently labelled lipid (DiO) and polymer $\left(\mathrm{PBd}_{22}-\right.$ $\mathrm{PEO}_{14}-\mathrm{TMR}$ ) were measured for each GUV. In Figure 1, a normalised relative intensity ratio is measured for these two probes, where a value tending towards 1 indicates a higher relative polymer dye intensity within the membrane and hence a polymer-rich vesicle, while values tending towards 1 indicate a higher relative lipid dye intensity, implying a lipid-rich membrane. A relative intensity ratio of 0 indicates the ideal mixing of the lipids and polymers in hybrid vesicles at the expected molar ratio for that sample (see the Experimental section for a more detailed description of this analysis).

Example images of $25 \mathrm{~mol} \% \mathrm{PBd}_{12}-\mathrm{PEO}_{11}$ GUVs in Figure 1 show the separate $\mathrm{DiO}$ and $\mathrm{PBd}_{22}-\mathrm{PEO}_{14}$ $-\mathrm{TMR}$ channels as well as the merged image. The images of $\mathrm{DiO}$ and $\mathrm{PBd}_{22}-\mathrm{PEO}_{14}-\mathrm{TMR}$ channels in other hybrid compositions can be found in the ESI. While both dyes are present in all GUVs, it is visually apparent that there are relative composition differences, where the $\mathrm{DiO}$ or $\mathrm{PBd}_{22}-\mathrm{PEO}_{14}-$ TMR probes are relatively brighter in individual GUVs, indicating the presence of lipid-rich (red arrows) and polymer-rich (blue arrows) GUVs, respectively.

As shown by

and Figure 1a, the relative intensity ratios of hybrid $\mathrm{PBd}_{22}-\mathrm{PEO}_{14} \mathrm{GUVs}$ have a monomodal distribution centred close to 0 , indicating most GUVs are well mixed hybrid membranes, containing the expected average compositions of both the $\mathrm{DiO}$ and $\mathrm{PBd}_{22}-\mathrm{PEO}_{14}-\mathrm{TMR}$ probes. This data also indicates that there are no populations of pure $\mathrm{PBd}_{22}-\mathrm{PEO}_{14}$ polymer or pure POPC lipid vesicles in these samples. However the distributions are broad, indicating a significant range in individual GUV compositions centred around the mean composition of the sample.

A similar phenomenon is observed for $\mathrm{PBd}_{12}-\mathrm{PEO}_{11}$ hybrid GUVs in Figure $1 \mathrm{~b}$. The shorter $\mathrm{PBd}_{12^{-}}$ $\mathrm{PEO}_{11}$ polymer is closer to the lipids in $\mathrm{Mw}$ but still a broad monomodal distribution is observed centred on an evenly mixed GUV composition. Of note, $50 \mathrm{~mol} \% \mathrm{PBd}_{12}-\mathrm{PEO}_{11}$ hybrid GUVs exhibited the broadest distribution in relative intensity ratio, with a broader tail towards polymer-rich compositions. This suggests that near equimolar lipid-polymer hybrid GUVs might be less favourable to form, when compared to the lipid-rich $25 \mathrm{~mol} \%$ and polymer-rich $75 \mathrm{~mol} \%$ compositions. This is analogous to early work on POPC/PBd $46-\mathrm{PEO}_{30}$ hybrid vesicles, where GUVs of intermediate lipid/polymer composition ratios could not be formed by electroformation. ${ }^{13}$ However, there is no evidence of enhanced heterogeneity in $50 \mathrm{~mol} \%$ hybrid GUVs formed with the intermediate size $\mathrm{PBd}_{22}-\mathrm{PEO}_{14}$ polymer (Figure 1a).

Table 1: The mean ( \pm standard error in mean) and variance of the overlayed distribution curves displayed in Figure 1.

\begin{tabular}{|c|c|c|}
\hline Sample & Normal Distribution Mean & Normal Distribution Variance \\
\hline $75 \mathrm{~mol} \% \mathrm{PBd}_{22}-\mathrm{PEO}_{14}$ & $0.009 \pm 0.008$ & 0.027 \\
\hline $50 \mathrm{~mol} \% \mathrm{PBd}_{22}-\mathrm{PEO}_{14}$ & $0.016 \pm 0.009$ & 0.023 \\
\hline $25 \mathrm{~mol} \% \mathrm{PBd}_{22}-\mathrm{PEO}_{14}$ & $0.039 \pm 0.008$ & 0.028 \\
\hline $75 \mathrm{~mol} \% \mathrm{PBd}_{12}-\mathrm{PEO}_{11}$ & $-0.014 \pm 0.007$ & 0.027 \\
\hline $50 \mathrm{~mol} \% \mathrm{PBd}_{12}-\mathrm{PEO}_{11}$ & $0.015 \pm 0.008$ & 0.038 \\
\hline $25 \mathrm{~mol} \% \mathrm{PBd}_{12}-\mathrm{PEO}_{11}$ & $-0.0003 \pm 0.005$ & 0.015 \\
\hline
\end{tabular}




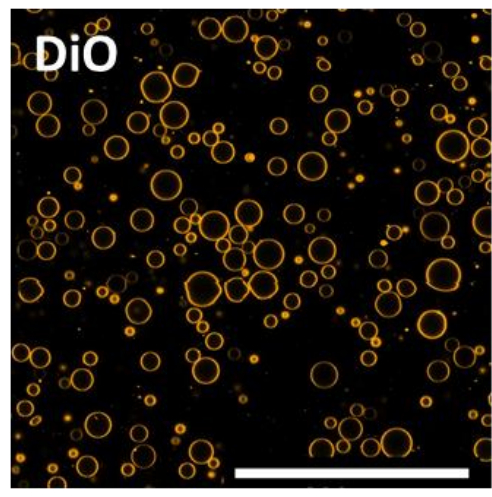

a)

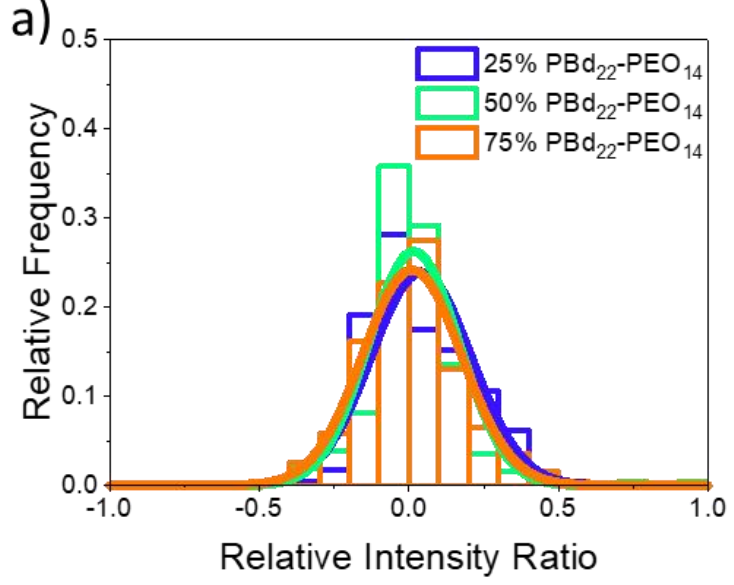

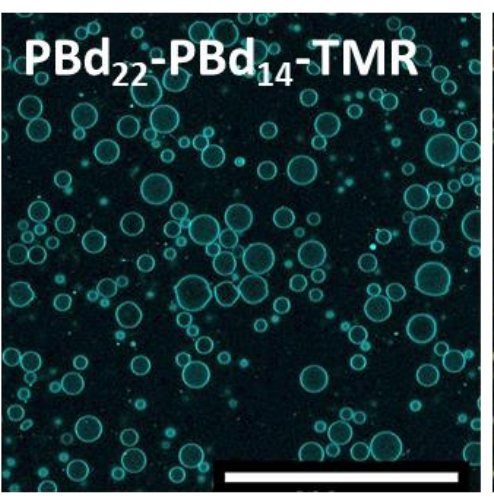

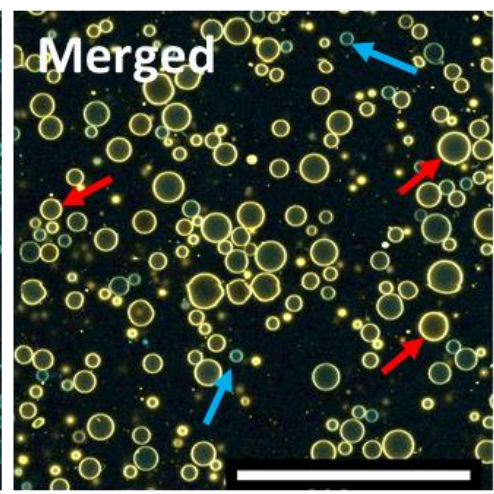

b)

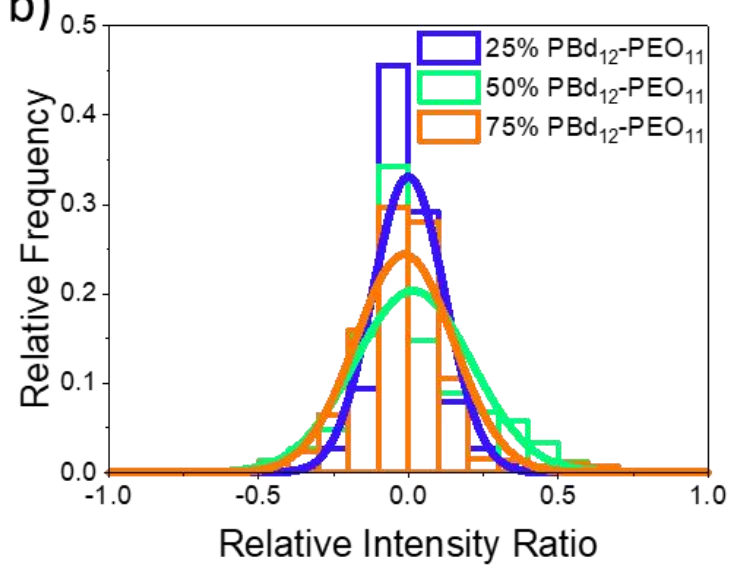

Figure 1: Images of intensity contributions from $\mathrm{DiO}$ and, $\mathrm{PBd}_{22}-\mathrm{PEO}_{14}-\mathrm{TMR}$ dyes individually in $25 \mathrm{~mol}^{2} \mathrm{PBd}_{12}-$ $\mathrm{PEO}_{11}$ GUVs, and the merged channels. Red arrows indicate vesicles with greater DiO contribution, while blue arrows indicate vesicles with greater $\mathrm{PBd}_{22}-\mathrm{PEO}_{14}-\mathrm{TMR}$ contribution to the overall fluorescence intensity of the GUV. The histograms show the relative intensity contributions from DiO and $\mathrm{PBd}_{22}-\mathrm{PEO}_{14-}-\mathrm{TMR}$ in a) $\mathrm{PBd}_{22}-$ $\mathrm{PEO}_{14} / \mathrm{POPC}$ and $\mathrm{b}$ ) $\mathrm{PBd}_{12}-\mathrm{PEO}_{11} / \mathrm{POPC}$ hybrid vesicles. An intensity ratio of 0 indicates equal relative fluorescence intensity contributions in hybrid vesicles from both $\mathrm{PBd}_{22}-\mathrm{PEO}_{14}-\mathrm{TMR}$ and DiO. Scale bars indicate $200 \mu \mathrm{m}$.

As the two fluorophores present in the hybrid GUVs have overlapping emission and excitation spectra, FRET from the lipid fluorophore, DiO, to the polymer $\mathrm{PBd}_{22}-\mathrm{PEO}_{14}-\mathrm{TMR}$ probe could occur. This would decrease the fluorescence intensity from the lipid DiO, potentially shifting the relative intensity ratio towards 1 and resulting in the observation of apparently polymer-rich membranes. This effect is not seen in Figure 1, likely due to a low FRET efficiency and the normalisation of DiO intensity in the analysis of these images. Sequential imaging of the two fluorophores means that FRET-enhanced fluorescence of the $\mathrm{PBd}_{22}-\mathrm{PEO}_{14}-\mathrm{TMR}$ probe would not be observed. Therefore our experimental design negates any potential effects from FRET between these probes that could impact our results and interpretation.

\section{Membrane hydration of hybrid GUVs is minimally affected by polymer composition}

Changes in membrane hydration with increasing polymer fraction in $\mathrm{PBd}_{22}-\mathrm{PEO}_{14} / \mathrm{POPC}$ and $\mathrm{PBd}_{12}-$ $\mathrm{PEO}_{11} / \mathrm{POPC}$ vesicle compositions was monitored using the fluorescent probe Laurdan ${ }^{24-27}$ Previous studies have used the shift in Laurdan fluorescence emission between $444 \mathrm{~nm}$ and $488 \mathrm{~nm}$ to calculate a General Polarisation (GP) value that can be a quantitative measure for lipid packing. These studies have shown that lipids in the fluid phase are more hydrated, while lipids in the gel phase have more restricted motion, and are less hydrated..$^{24} \mathrm{GP}$ values close to 1 indicate a highly ordered environment 
with little to no hydration, while a GP towards -1 indicates Laurdan is in a well hydrated, disordered environment.

Laurdan-labelled GUVs were used to obtain GP values from 15-20 individual vesicles for each GUV composition studied using a confocal microscope. Interestingly, all compositions studied (pure lipid and pure polymer vesicles and $50 \mathrm{~mol} \%$ hybrid GUVs) were found to have GP values $<0$, indicating a hydrated disordered membrane (Figure 2). There is only a small apparent increase in the average GP value between the pure lipid GUVs and the GUVs made purely of each polymer (Figure 2a), where
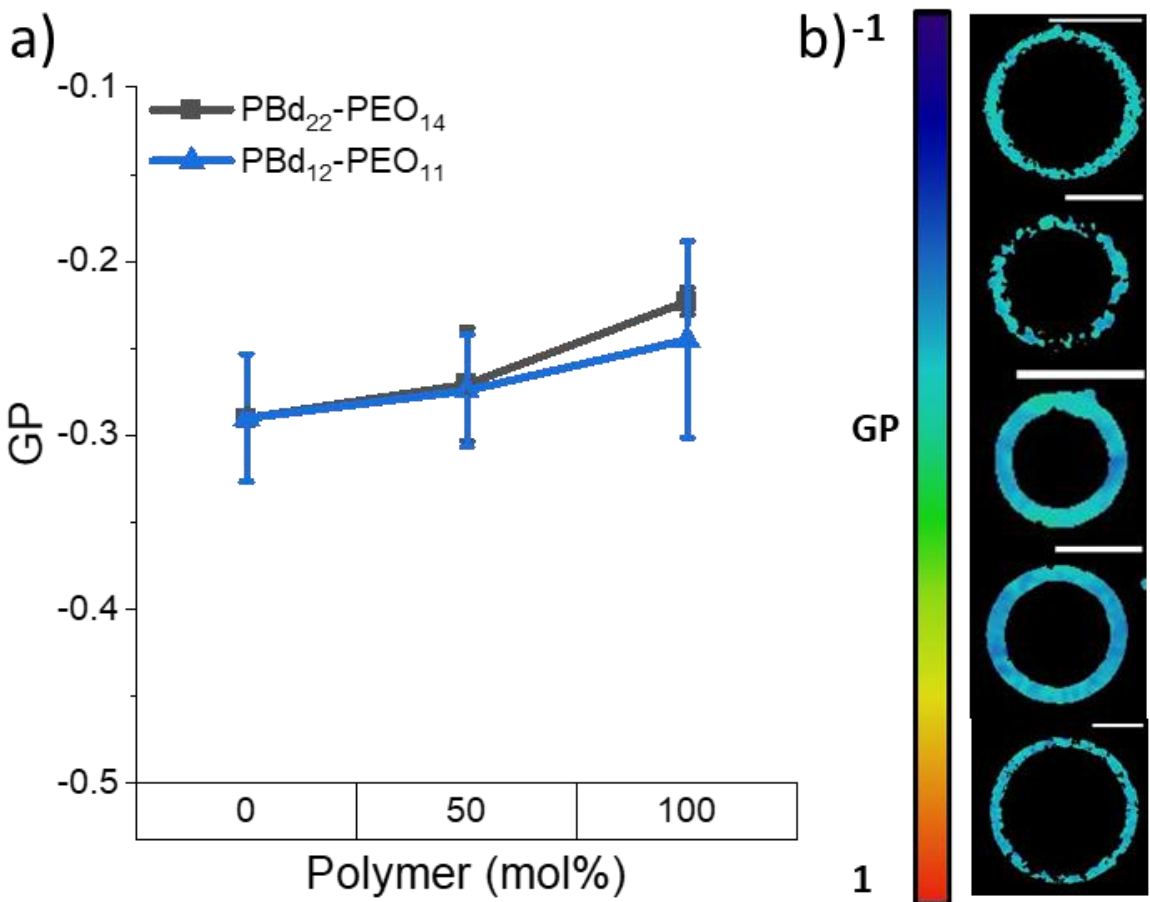

$$
100 \%
$$$$
\mathrm{PBd}_{22} \mathrm{PEO}_{14}
$$

$50 \%$

$\mathrm{PBd}_{22} \mathrm{PEO}_{14}$

POPC

$50 \%$

$\mathrm{PBd}_{12} \mathrm{PEO}_{11}$

$100 \%$

$\mathrm{PBd}_{12} \mathrm{PEO}_{11}$

Figure 2: a) GP values of GUVs as polymer fraction increases in $\mathrm{PBd}_{22}-\mathrm{PEO}_{14}$ and $\mathrm{PBd}_{12}-\mathrm{PEO}_{11}$ vesicles. Negative GP values indicate a disordered membrane, while positive GP values indicate an ordered membrane. b) Colour maps indicating GP values in example GUV images at each composition. The black spots on the colour map indicate no spectral data at those points. All spectral images can be found in the ESI. Scale bars represent $10 \mu \mathrm{m}$.

the measured difference in GP is not statistically significant $(\mathrm{p}>0.05$, Tukey and Bonferroni

ANOVA). Therefore all hybrid GUV membranes studied here have a comparable hydration state and membrane ordering, which likely contributes to the formation of well-mixed hybrid GUVs. No lateral heterogeneities were observed in the hydration state of these membranes, providing further evidence for a lack of distinct microscale membrane domains forming in these GUV compositions (Figure 2b). The spectral images at $444 \mathrm{~nm}$ and $488 \mathrm{~nm}$ used to calculate the GP can be found in the ESI.

\section{Membrane diffusion fluidity decreases with increasing polymer fraction}

While structurally, the hydration state of lipid-rich and polymer-rich GUV membranes are comparable, this does not inform us about the dynamics within these different membrane compositions. We therefore investigate hybrid membrane dynamics using fluorescence recovery after photobleaching (FRAP). The DiO lipid probe and the $\mathrm{PBd}_{22}-\mathrm{PEO}_{14}-\mathrm{TMR}$ polymer probe were used to independently study the mobility of lipid-like and polymer-like molecules in these membrane environments, respectively. The diffusion coefficients shown in Figure 3 were extrapolated from the fluorescence recovery curves of a bleached area in the upper pole of a vesicle. The fluorescence recovery curves were fitted using modified Bessel functions with a single recovery time. ${ }^{28}$ Attempts to fit the recovery data with two recovery times did not improve the fits, indicating that the probes 
diffuse through a homogeneous membrane environment, which is supported by our measurements of hybrid membrane mixing and hydration state that we have already presented.

The DiO lipid probe was included in all lipid containing GUV compositions (0-75 mol\% polymer) and is assumed to be representative of lipid diffusion in a hybrid membrane. The $\mathrm{DiO}$ probe diffusion coefficient decreased with increasing polymer fraction from $3.31 \pm 0.56 \mu \mathrm{m} \mathrm{s}^{-1}$ for single component POPC GUVs ( $0 \mathrm{~mol} \%$ polymer) to $0.46 \pm 0.15 \mu \mathrm{m} \mathrm{s}^{-1}$ in $75 \mathrm{~mol} \% \mathrm{PBd}_{22}-\mathrm{PEO}_{14}$ hybrid vesicles and $0.68 \pm 0.23 \mu \mathrm{m} \mathrm{s}^{-1}$ in $75 \mathrm{~mol} \% \mathrm{PBd}_{12}-\mathrm{PEO}_{11}$ hybrid vesicles. In $\mathrm{PBd}_{22}-\mathrm{PEO}_{14}$ vesicle compositions, only the reduction in $\mathrm{DiO}$ diffusion between $0 \mathrm{~mol} \%$ and $25 \mathrm{~mol} \% \mathrm{PBd}_{22}-\mathrm{PEO}_{14}$ is statistically significant in nearest neighbour compositions. However, next-nearest neighbour and wider spread compositions did show statistically significant differences $(\mathrm{p}<0.05$, Tukey and Bonferroni ANOVA) in diffusion coefficient (comparing 0 mol\% polymer with 50 and $75 \mathrm{~mol} \%$ vesicles and $25 \mathrm{~mol} \%$ with $75 \mathrm{~mol} \%$ vesicles). For all $\mathrm{PBd}_{12}-\mathrm{PEO}_{11}$ hybrid vesicle compositions, the differences in $\mathrm{DiO}$ diffusion is statistically significant $(\mathrm{p}<0.05)$. The large, significant drop in DiO diffusion coefficient between 0 $\mathrm{mol} \%$ and $25 \mathrm{~mol} \% \mathrm{PBd}_{22}-\mathrm{PEO}_{14} \mathrm{GUVs}$ suggests that a minority component of these larger polymers in hybrid membranes can have a dominant influence on the fluidity of the composite membrane.

The diffusion of polymers through the structural matrix of hybrid membranes was monitored using the fluorescently labelled polymer, $\mathrm{PBd}_{22}-\mathrm{PEO}_{14}-\mathrm{TMR}$. This probe was incorporated in block copolymer containing membrane compositions (25-100 mol\% polymer). The diffusion coefficient of $\mathrm{PBd}_{22}-\mathrm{PEO}_{14}$-TMR decreased with increasing polymer fraction, from $0.75 \pm 0.25 \mu \mathrm{ms}^{-1}$ for $25 \mathrm{~mol} \%$ $\mathrm{PBd}_{22}-\mathrm{PEO}_{14}$ vesicles to $0.67 \pm 0.28 \mu \mathrm{ms}^{-1}$ in $100 \mathrm{~mol} \%$ (single component) $\mathrm{PBd}_{22}-\mathrm{PEO}_{14}$ hybrid vesicles and $1.67 \pm 0.52 \mu \mathrm{ms}^{-1}$ in $25 \mathrm{~mol} \% \mathrm{PBd}_{12}-\mathrm{PEO}_{11}$ hybrid vesicles to $0.71 \pm 0.56 \mu \mathrm{ms}^{-1}$ in 100 mol\% $\mathrm{PBd}_{12}-\mathrm{PEO}_{11}$ vesicles. Although the measured polymer diffusion through the hybrid membranes decreased with increasing polymer fraction in $\mathrm{PBd}_{22}-\mathrm{PEO}_{14}$ hybrid vesicles, this is not statistically significant between any pairwise comparison of compositions. This suggests that the polymer mobility is minimally affected by the lipid composition within the range studied. For $\mathrm{PBd}_{12}-\mathrm{PEO}_{11}$ hybrid compositions, the decrease between neighbouring compositions is only statistically significant $(\mathrm{p}<0.05$, Tukey and Bonferroni ANOVA) for the nearest neighbour compositions except between 50 mol\% and $75 \mathrm{~mol} \% \mathrm{PBd}_{12}-\mathrm{PEO}_{11}$. Next nearest neighbour comparisons where significant (comparison of $25 \mathrm{~mol} \%$ with $75 \mathrm{~mol} \%$ and $100 \mathrm{~mol} \% \mathrm{PBd}_{12}-\mathrm{PEO}_{11}$ membranes, and $50 \mathrm{~mol} \%$ with $100 \mathrm{~mol} \%$. This shows that for the smaller Mw polymer, the lipid composition has a greater impact on its mobility within the membrane than for the larger polymer.

Overall, the diffusion of the smaller lipid-like DiO probe is faster than that of the larger $\mathrm{PBd}_{22}-\mathrm{PEO}_{14^{-}}$ TMR probe, which would be expected due to the reduced free volume required for the smaller lipid probe to hop laterally between sites in the membrane matrix. The absolute fluorescence recovery of both the lipid and polymer probes also decreases with decreased membrane fluidity: this is likely caused by enhanced photobleaching of these probes in less fluid matrices, resulting in enhanced total bleaching withing the finite system size of a single GUV. 
a)

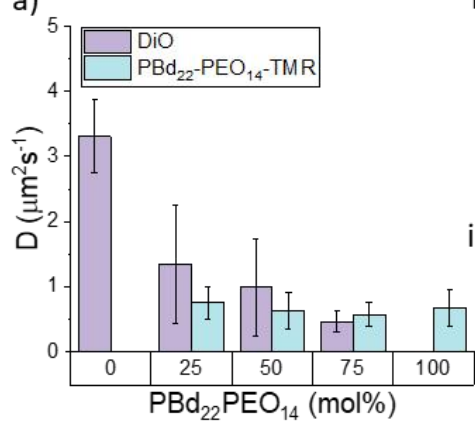

c)

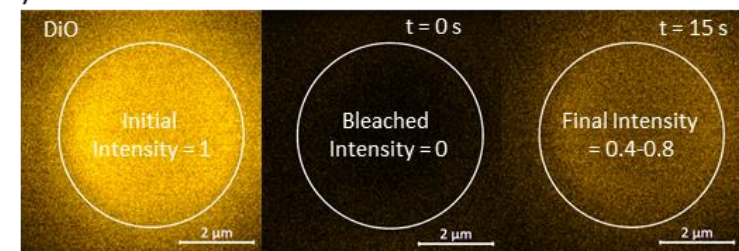

b)
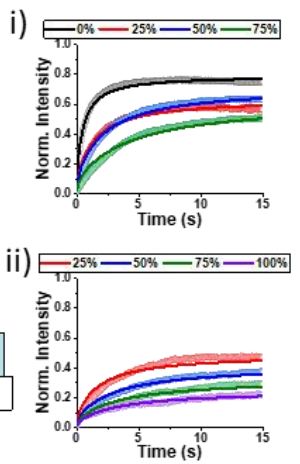
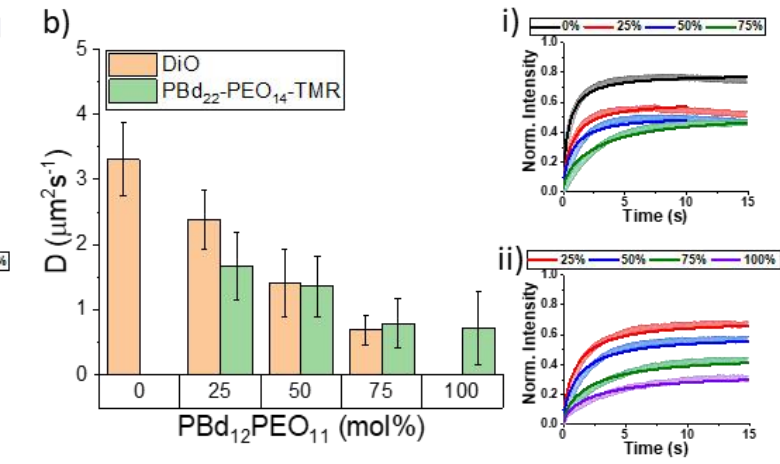

d)

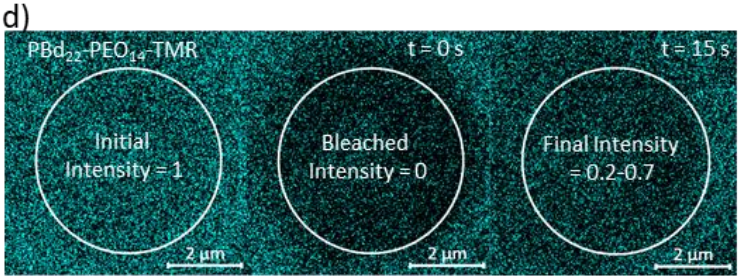

Figure 3: The diffusion coefficients of a) $\mathrm{PBd}_{22}-\mathrm{PEO}_{14}$ vesicles and b) $\mathrm{PBd}_{12}-\mathrm{PEO}_{11}$ vesicles as well as the fluorescence recovery after photobleaching profiles from i) $\mathrm{DiO}$ and ii) $\mathrm{PBd}_{22}-\mathrm{PEO}_{14}-\mathrm{TMR}$ fluorescent dyes in each composition. Images of the bleaching process of c) $\mathrm{DiO}$ and d) $\mathrm{PBd}_{22}-\mathrm{PEO}_{14}-\mathrm{TMR}$ shows fluorescence intensity is not fully recovered.

\section{Conclusion}

The lipid POPC and PBd-PEO polymers were blended into mixtures to form hybrid GUVs. As polymer molecular weight is thought to control the membrane thickness and therefore the membrane's bulk properties, ${ }^{29} \mathrm{PBd}-\mathrm{PEO}$ polymers of two different sizes $\left(\mathrm{PBd}_{22}-\mathrm{PEO}_{14}\right.$ or $\left.\mathrm{PBd}_{12}-\mathrm{PEO}_{11}\right)$ were compared. Hybrid vesicles were formed with well-mixed and homogenous membranes with a monomodal but broad composition distribution between individual GUVs. No evidence for lipid-rich or polymer-rich microscopic membrane domains was observed. The homogeneity and mixing of these hybrid GUV membranes was also supported by Laurdan GP measurements, which showed comparable hydration states between the lipid-only and polymer-only GUV compositions. This provides further support for the apparent compatibility of PBd-PEO polymers with fluid phase phospholipids like POPC.

Fluorophore diffusion measurements provide information on the viscosity and dynamics in hybrid membranes. The diffusion of both the lipid-like $\mathrm{DiO}$ probe and $\mathrm{PBd}_{22}-\mathrm{PEO}_{14}-\mathrm{TMR}$ probe decrease with increasing block copolymer content in the GUVs. The smaller lipid probe diffuses faster than the polymer probe, as would be anticipated, and the membranes containing the shorter $\mathrm{PBd}_{12}-\mathrm{PEO}_{11}$ polymer also facilitate faster lateral diffusion in the membrane. The decrease in lateral diffusion is not directly proportional to the polymer composition in the membrane. Notably, the diffusion in $\mathrm{PBd}_{22}-$ $\mathrm{PEO}_{14}$-containing mixtures is strongly impacted by a minority composition of the polymer in the membrane.

This work demonstrates that hybrid phospholipid/PBd-PEO vesicles form mixed composite systems with tuneable membrane structure and dynamics, which can now be optimised for specific vesicle biotechnology applications. 


\section{Experimental}

\section{Materials}

1-palmitoyl-2-oleoyl-sn-glycero-3-phosphocholine (POPC) was purchased from Avanti Polar Lipids Inc. (Alabaster, AL). Diblock copolymer, poly(1,2-butadiene-block-ethylene oxide) (PBd-PEO) of two different molecular weights $\left(\mathrm{PBd}_{22}-\mathrm{PEO}_{14}, \mathrm{Mr}=1800 \mathrm{~g} \cdot \mathrm{mol}^{-1}\right.$ and $\mathrm{PBd}_{12}-\mathrm{PEO}_{11}, \mathrm{Mr}=1150$ g. $\mathrm{mol}^{-1}$ ) were purchased from Polymer Source, Inc. (Montreal, Canada). $\mathrm{PBd}_{22}-\mathrm{PEO}_{14}$ (PDI 1.01) has a hydrophobic PBd block of 1200 g.mol ${ }^{-1}$ (> 85\% 1,2 addition) and a hydrophilic PEO block of 600 g. $\mathrm{mol}^{-1}$, while $\mathrm{PBd}_{12}-\mathrm{PEO}_{11}$ (PDI 1.09) has a hydrophobic PBd block of 650 g.mol ${ }^{-1}$ (> 85\% 1,2 addition) and a hydrophilic PEO block of 500 g.mol ${ }^{-1}$. $\mathrm{PBd}_{22}-\mathrm{PEO}_{14}$ block copolymer was labelled with tetramethyl rhodamine (TMR) to synthesise $\mathrm{PBd}_{22}-\mathrm{PEO}_{14}$-TMR following the procedure described in the ESI. Characterisation by LC-MS, IR spectroscopy and COSY-HNMR spectroscopy is also provided in the ESI.

6-dodecanoyl-2-dimethylaminonaphthalene (Laurdan), was obtained from Sigma-Aldrich (Gillingham, U.K.). $\mathrm{DiOC}_{18}$ (3) (3,3'-dioctadecyloxacarbocyanine perchlorate) (DiO) was purchased from ThermoFisher Scientific Ltd. (Loughborough, Leicestershire, U.K.).

\section{Preparation of giant unilamellar vesicles}

Hybrid GUVs were prepared using the electroformation method from $6.57 \mathrm{mM}$ POPC and PBd-bPEO solutions in various polymer-to-lipid ratios, with either $2 \mathrm{~mol} \% \mathrm{DiO}$ and $10 \mathrm{~mol} \% \mathrm{PBd}_{22}-\mathrm{PEO}_{14}$ TMR for FRAP and lipid/polymer mixing ratio experiments, or $0.5 \mathrm{~mol} \%$ Laurdan for membrane hydration measurements. True mole fractions of POPC, PBd-PEO, DiO and $\mathrm{PBd}_{22}-\mathrm{PEO}_{14}-\mathrm{TMR}$ in $\mathrm{PBd}_{22}-\mathrm{PEO}_{14}$ and $\mathrm{PBd}_{12}-\mathrm{PEO}_{11}$ GUV compositions are provided in the ESI.

Briefly, $9 \mu \mathrm{L}$ of a lipid/polymer solution was deposited as a thin layer over the conductive side of each of the two indium-tin oxide (ITO)-coated glass slides with resistivity of 8-12 $\Omega \mathrm{m}^{-2}$ and dried. The ITO slides were then assembled into an electroformation chamber each in contact with copper tape and separated by a Teflon spacer. The chamber was filled with a $300 \mathrm{mM}$ sucrose solution (300 mOsmkg ${ }^{-1}$ measured using a 3320 single-sample micro-osmometer (Advanced Instruments, Norwood, U.S.A.)) and connected to an arbitrary function generator 1022 (Tektronix, Bracknell, U.K.) to apply an alternating current electric field.

A sine wave of frequency $10 \mathrm{~Hz}$ was used for electroformation at different temperatures depending on the membrane composition with the peak to peak voltage changing every 10 minutes from 0.1 to 0.5 , 1,2 and finally $3 \mathrm{~V}$ for 2 hours. $0-50 \mathrm{~mol} \% \mathrm{PBd}_{12}-\mathrm{PEO}_{11}$, electroformation was carried out at $35^{\circ} \mathrm{C}$, 25 and $50 \mathrm{~mol} \% \mathrm{PBd}_{22}-\mathrm{PEO}_{14}$ at $42{ }^{\circ} \mathrm{C}$, and 75 and $100 \mathrm{~mol} \%$ of both polymers at $64{ }^{\circ} \mathrm{C}$. The frequency was gradually decreased over approximately $8 \mathrm{~min}$ to facilitate the closure and detachment of GUVs from the surface. After electroformation, the GUVs were suspended in an isoosmolar 20 $\mathrm{mM}$ HEPES, $150 \mathrm{mM} \mathrm{NaCl}$ solution (confirmed by measurement using the micro-osmometer).

Microscopy studies were conducted at room temperature on a Zeiss LSM880 + Airyscan inverted confocal microscope. The samples were deposited on the microscope slides previously treated with a $5 \%$ BSA solution to prevent GUVs from adhering and rupturing on the glass.

\section{Determination of polymer-to-lipid relative intensity ratio}

Changes in intensity of $\mathrm{DiO}$ and $\mathrm{PBd}_{22}-\mathrm{PEO}_{14}-\mathrm{TMR}$ dyes in hybrid GUV membranes was monitored with a Zeiss LSM880 + Airyscan inverted confocal microscope on the same day of GUV formation. The microscope tile scanning option with a $2 \%$ intensity laser beam (488 $\mathrm{nm}$ for DiO excitation and 
$561 \mathrm{~nm}$ for $\mathrm{PBd}_{22}-\mathrm{PEO}_{14}-\mathrm{TMR}$ excitation) and pinhole aperture of $0.7 \mu \mathrm{m}$ was used to scan large areas of the sample and facilitate the acquisition of statistical data. The images were analysed using FIJI software (National Institutes of Health, Bethesda, MD).

The image was split into channels, representing the intensity contribution from each fluorescent dye. The image from the $\mathrm{PBd}_{22}-\mathrm{PEO}_{14}-\mathrm{TMR}$ channel was duplicated to create the mask. First this duplicate was adjust for brightness and contrast, and a threshold applied. Then a mask was created to ensure only vesicles greater than $>10 \mu \mathrm{m}$ were measured using the automatic particle analysis tool. This size was chosen to prevent selection of non-vesicular aggregates. The mask created regions of interest around selected vesicles of a particular size. These regions of interest were then applied to the split channel images with intensity contributions from each dye to retrieve their intensity values. The intensity values from each dye image are normalised to the mean. Then the relative intensity ratio, $R$, between the intensity contributions from $\mathrm{DiO}, \mathrm{I}_{\mathrm{DiO}}$, and $\mathrm{PBd}_{22}-\mathrm{PEO}_{14}-\mathrm{TMR}, \mathrm{I}_{\mathrm{PBd} 22-\mathrm{PEO} 14-\mathrm{TMR}}$, was calculated using:

$$
\mathrm{R}=\frac{I_{P B d 22-P E O 14-T M R}-I_{D i O}}{I_{P B d 22-P E O 14-T M R}+I_{D i O}}
$$

Equation 1

This gives a normalised value between -1 and 1 , where -1 indicates the intensity contributions in hybrid vesicles are from $\mathrm{DiO}$ only, 1 where the intensity contributions in the hybrid vesicles are entirely from $\mathrm{PBd}_{22}-\mathrm{PEO}_{14}-\mathrm{TMR}$, while an intensity ratio of 0 indicates equal fluorescence intensity contributions in hybrid vesicles from both $\mathrm{PBd}_{22}-\mathrm{PEO}_{14}-\mathrm{TMR}$ and $\mathrm{DiO}$. The resultant histograms are then fitted with a normal distribution curve.

\section{Spectral Imaging of Laurdan}

Spectral imaging of the different GUV compositions was performed using the Zeiss LSM880 + Airyscan inverted confocal microscope 32-channel GaAsP detector array. Laurdan is excited at 405 $\mathrm{nm}$ using a $4 \%$ laser intensity. The lambda detection range was set between 412 and $555 \mathrm{~nm}$ with intervals of $8.9 \mathrm{~nm}$, allowing simultaneous coverage of the whole emission spectrum. The confocal pinhole aperture was set to $3.1 \mu \mathrm{m}$ and, to increase the scan speed, single GUVs were maximally magnified to allow the whole vesicle to be imaged. The images of resolution $512 \times 512$ pixels were saved in 'lsm' file format and filtered with a Gaussian blur filter before being analysed to find the general polarisation value of each pixel using a custom plug-in compatible with Fiji, found at https://github.com/dwaithe/GP-plugin. ${ }^{26}$

By recording the whole emission spectrum for each image pixel, the spatial heterogeneity in GP values can be accurately observed. The whole emission spectrum of Laurdan for each image pixel is recorded and a Gaussian distribution was fitted to the emission spectrum captured for each pixel. From this, the GP value for each pixel was obtained by comparing the fluorescence signal intensities at liquid disordered or liquid ordered emission wavelengths. For Laurdan, emission at wavelength 444 $\mathrm{nm}$ (blue-shifted) indicates an ordered membrane environment, while emission at $488 \mathrm{~nm}$ (redshifted) indicates a disordered membrane environment ${ }^{26}$ :

$$
\mathrm{GP}=\frac{I_{444 \mathrm{~nm}}-I_{488 \mathrm{~nm}}}{I_{444 \mathrm{~nm}}+I_{488 \mathrm{~nm}}}
$$

\section{Fluorescence recovery after photobleaching (FRAP)}

FRAP consists of irreversibly bleaching fluorophores in a particular region of interest (ROI). To do this, the ROI was imaged at 100\% intensity for all lasers $(405,458,514,561$ and $633 \mathrm{~nm})$. The fluorescence recovery is then monitoring with a $2 \%$ intensity laser $(488 \mathrm{~nm}$ for DiO excitation and 
$561 \mathrm{~nm}$ for $\mathrm{PBd}_{22}-\mathrm{PEO}_{14}-\mathrm{TMR}$ excitation) and a confocal pinhole aperture of $3.1 \mu \mathrm{m}$. The rate of fluorescence recovery that represents the time needed for the fluorophores in the membrane that surround the ROI to diffuse into that region, re-equilibrating the local fluorescence intensity. FRAP experiments were performed on the upper pole of GUVs (away from the coverslip), where a circular region of interest with a $5 \pm 0.5 \mu \mathrm{m}$ diameter was exposed to five bleaching scans at $100 \%$ laser power; the recovery was then monitored by recording a time series at a frame rate of 13 frames per second of size $256 \times 256$ pixels. The recovery curves were fitted with Origin Pro using the fluorescence recovery model of Soumpasis ${ }^{28,30}$ :

$$
\mathrm{f}(\mathrm{t})=\mathrm{A}\left\{\exp \left(-\frac{2 \tau_{\mathrm{D}}}{\mathrm{t}}\right)\left[\mathrm{J}_{0}\left(\frac{2 \tau_{\mathrm{D}}}{\mathrm{t}}\right)+\mathrm{J}_{1}\left(\frac{2 \tau_{\mathrm{D}}}{\mathrm{t}}\right)\right]\right\}
$$

Equation 3

where $t$ is the time, $A$ is the recovery level, $\tau_{D}$ is the half recovery time, and $J_{0}$ and $J_{1}$ are modified Bessel functions of the first kind. The diffusion coefficient, D, can then be calculated from the recovery times and the radius of the bleached region, $r$, using:

$$
\mathrm{D}=\frac{\mathrm{r}^{2}}{4 \tau_{\mathrm{D}}}
$$

The fluorescence recovery data was modelled using a single diffusion coefficient for all membrane compositions. Between 15 and 20 GUVs were analysed for each composition and the values averaged.

\section{Author Contributions}

R. Seneviratne: conceptualisation, investigation, formal analysis and writing of initial draft. M. Rappolt \& L. J. C. Jeuken: supervision and writing. P. A. Beales: conceptualisation, supervision, and writing.

\section{Conflicts of interest}

There are no conflicts to declare.

\section{Acknowledgements}

Jason Rowley, David Martin and Dr Andrew Booth for support creating the polymer dye. Dr Andrew Booth and Marcos Arribas Perez for assistance on confocal microscopy and fluorescence techniques.

\section{Notes and references}

1. E. Rideau, R. Dimova, P. Schwille, F. R. Wurm and K. Landfester, Chemical society reviews, 2018, 47, 8572-8610.

2. K. T. Powell and J. C. Weaver, Bioelectrochemistry and Bioenergetics, 1986, 15, 211-227.

3. A. A. Gurtovenko and I. Vattulainen, Biophysical journal, 2007, 92, 1878-1890.

4. $\quad$ S. Marrink, F. Jähnig and H. Berendsen, Biophysical journal, 1996, 71, 632-647.

5. C. G. Palivan, R. Goers, A. Najer, X. Zhang, A. Car and W. Meier, Chemical society reviews, 2016, 45, 377-411.

6. M. Chemin, P.-M. Brun, S. Lecommandoux, O. Sandre and J.-F. Le Meins, Soft Matter, 2012, 8, 2867-2874.

7. T. P. T. Dao, F. Fernandes, E. Ibarboure, K. Ferji, M. Prieto, O. Sandre and J.-F. Le Meins, Soft Matter, 2017, 13, 627-637.

8. S. Khan, M. Li, S. P. Muench, L. J. C. Jeuken and P. A. Beales, Chemical Communications, 2016, 52, 11020-11023.

9. S. Rottet, S. Iqbal, P. A. Beales, A. Lin, J. Lee, M. Rug, C. Scott and R. Callaghan, Polymers, 2020, 12, 1049.

10. C. Kleineberg, C. Wölfer, A. Abbasnia, D. Pischel, C. Bednarz, I. Ivanov, T. Heitkamp, M. Börsch, K. Sundmacher and T. Vidaković-Koch, ChemBioChem, 2020, 21, 2149-2160. 
11. W. F. Paxton, P. T. McAninch, K. E. Achyuthan, S. H. R. Shin and H. L. Monteith, Colloids and Surfaces B-Biointerfaces, 2017, 159, 268-276.

12. N. Hamada, S. Gakhar and M. L. Longo, Biochimica et Biophysica Acta (BBA)Biomembranes, 2021, 1863, 183552.

13. J. Nam, P. A. Beales and T. K. Vanderlick, Langmuir, 2011, 27, 1-6.

14. J. Nam, T. K. Vanderlick and P. A. Beales, Soft Matter, 2012, 8, 7982-7988.

15. C. Magnani, C. Montis, G. Mangiapia, A. F. Mingotaud, C. Mingotaud, C. Roux, P. Joseph, D. Berti and B. Lonetti, Colloids and Surfaces B: Biointerfaces, 2018, 168, 18-28.

16. S. Lim, H.-P. De Hoog, A. Parikh, M. Nallani and B. Liedberg, Polymers, 2013, 5, 11021114.

17. Z. Cheng, D. R. Elias, N. P. Kamat, E. D. Johnston, A. Poloukhtine, V. Popik, D. A. Hammer and A. Tsourkas, Bioconjugate chemistry, 2011, 22, 2021-2029.

18. J.-F. Le Meins, C. Schatz, S. Lecommandoux and O. Sandre, Materials today, 2013, 16, $397-$ 402.

19. R. Seneviratne, S. Khan, E. Moscrop, M. Rappolt, S. P. Muench, L. J. Jeuken and P. A. Beales, Methods, 2018, 147, 142-149.

20. M. L. Jacobs, M. A. Boyd and N. P. Kamat, Proceedings of the national academy of sciences, 2019, 116, 4031-4036.

21. T. T. Dao, F. Fernandes, M. Er-Rafik, R. Salva, M. Schmutz, A. Brûlet, M. Prieto, O. Sandre and J.-F. Le Meins, ACS Macro Letters, 2015, 4, 182-186.

22. A. F. Barton, Handbook of polymer-liquid interaction parameters and solubility parameters, Routledge, 2018.

23. A. F. Barton, CRC handbook of solubility parameters and other cohesion parameters, Routledge, 2017.

24. S. A. Sánchez, M. Tricerri, G. Gunther and E. Gratton, Modern research and educational topics in microscopy, 2007, 2, 1007-1014.

25. M. Amaro, F. Reina, M. Hof, C. Eggeling and E. Sezgin, Journal of physics D: Applied physics, 2017, 50, 134004.

26. E. Sezgin, D. Waithe, J. B. De La Serna and C. Eggeling, ChemPhysChem, 2015, 16, 1387.

27. K. Gaus, T. Zech and T. Harder, Molecular membrane biology, 2006, 23, 41-48.

28. D. Soumpasis, Biophysical journal, 1983, 41, 95-97.

29. C. LoPresti, H. Lomas, M. Massignani, T. Smart and G. Battaglia, Journal of Materials Chemistry, 2009, 19, 3576-3590.

30. D. Axelrod, D. Koppel, J. Schlessinger, E. Elson and W. W. Webb, Biophysical journal, 1976, 16, 1055-1069. 